\title{
Serum Carcinoembryonic Antigen Level Predicts Cancer- Specific Outcomes of Resected Non-Small Cell Lung Cancer With Interstitial Pneumonia
}

\author{
Masaki Tomita ${ }^{\mathrm{a}, \mathrm{c}}$, Takanori Ayabe ${ }^{\mathrm{a}}$, Ryo Maeda ${ }^{\mathrm{a}}$, \\ Kunihide Nakamura ${ }^{b}$
}

\begin{abstract}
Background: It has been well accepted that the prognosis of nonsmall cell lung cancer (NSCLC) patients with interstitial pneumonia (IP) is significantly poor. However, there are only a few studies that indicated the prognostic factors, especially tumor markers, among NSCLC patients with IP.

Methods: Forty-one NSCLC patients with IP who underwent surgery at our institution were included. Patients died of other diseases including postoperative acute exacerbation (AE) of IP were excluded. Univariate and multivariate analyses were calculated by the Cox proportional hazards regression model.
\end{abstract}

Results: The 5-year cancer-specific survival of overall and stage I patients were $37.4 \%$ and $39.2 \%$, respectively. The 5-year cancer-specific survival of patients with high serum carcinoembryonic antigen (CEA) level was 9.4\%, while that with normal serum CEA level was $55.6 \%$. However, serum cytokeratin-19 fragment (CYFRA 21-1) and squamous cell carcinoma-related antigen (SCC) levels were not associated with patients' survival. Furthermore, serum CEA level was significantly associated with poorer cancer-specific survival in univariate and multivariate analyses.

Conclusions: This study demonstrated that serum CEA level might serve as an efficient prognostic indicator after surgery in NSCLC with IP.

Keywords: Interstitial pneumonia; Non-small cell lung cancer; Tumor marker; CEA; Prognosis

Manuscript submitted October 15, 2018, accepted October 24, 2018

aDepartment of Thoracic and Breast Surgery, Faculty of Medicine, University of Miyazaki, Kihara 5200, Kiyotake, Miyazaki, 889-1692, Japan

bDepartment of Cardiovascular Surgery, Faculty of Medicine, University of Miyazaki, Kihara 5200, Kiyotake, Miyazaki, 889-1692, Japan

${ }^{\mathrm{c} C}$ Corresponding Author: Masaki Tomita, Department of Thoracic and Breast Surgery, Faculty of Medicine, University of Miyazaki, Kihara 5200, Kiyotake, Miyazaki, 889-1692, Japan. Email: mtomita@med.miyazaki-u.ac.jp

doi: https://doi.org/10.14740/wjon1163

\section{Introduction}

It has been well accepted that lung resection has been shown to be associated with high postoperative morbidity and mortality in non-small cell lung cancer (NSCLC) patients with interstitial pneumonias (IP) [1-3]. The acute exacerbation (AE) of IP after lung resection is most important comorbidity and is associated with mortality rates [1-3]. Sato et al examined the incidence, risk factors, and mortality rate of AE from 1,763 patients from 61 Japanese institutions [2]. They reported that AE occurred in $9.3 \%$ of patients, with a mortality rate of $43.9 \%$. In addition to postoperative $\mathrm{AE}$ occurrence, it has been reported that the long-term survival of NSCLC patients with IP is poor [2].

With regard to the long-term survival of NSCLC patients with IP after surgery, there are only a few studies that found the factors associated with the long-term cancer-specific survival of these patients. Sato et al also analyzed data from 1,763 NSCLC patients with IP, and they demonstrated that the surgical procedure (wedge resection vs. lobectomy) and a predicted percent vital capacity $(\% \mathrm{VC})$ less than $80 \%$ were associated with survival of NSCLC patients with IP [4]. Recently, Kobayashi et al [5] reported that the Glasgow prognostic score (GPS), which uses serum C-reactive protein (CRP) and albumin levels, is an independent prognostic factor of survival in NSCLC patients with IP undergoing surgical resection.

Many previous studies reported the prognostic significance of serum tumor markers, including serum carcinoembryonic antigen (CEA) [6, 7], cytokeratin-19 fragment (CYFRA 21-1) [8,9] and squamous cell carcinoma-related antigen (SCC) levels [10] in NSCLC undergoing surgical resection. To our knowledge, however, there are no previous studies that evaluated the prognostic significance of serum tumor markers in NSCLC patients with IP. Therefore, in the present study, we examined the prognostic significance of preoperative serum CEA, CYFRA 21-1 and SCC levels in NSCLC patients with IP.

\section{Patients and Methods}

This retrospective study examined the records of a sequential series of 41 patients with NSCLC with IP between January, 
Table 1. Clinical Characteristics of Patients

\begin{tabular}{|c|c|}
\hline & Number of patients \\
\hline \multicolumn{2}{|l|}{ Age } \\
\hline$<65$ & 16 \\
\hline$\geq 65$ & 25 \\
\hline \multicolumn{2}{|l|}{ Gender } \\
\hline Male & 31 \\
\hline Female & 10 \\
\hline \multicolumn{2}{|l|}{ Smoking status } \\
\hline Never & 8 \\
\hline Current/former & 33 \\
\hline \multicolumn{2}{|l|}{$\% \mathrm{VC}$} \\
\hline$<80$ & 5 \\
\hline$\geq 80$ & 36 \\
\hline \multicolumn{2}{|l|}{ UIP } \\
\hline UIP & 17 \\
\hline non-UIP & 24 \\
\hline \multicolumn{2}{|l|}{ Histology } \\
\hline Adenocarcinoma & 21 \\
\hline Squamous cell carcinoma & 14 \\
\hline Others & 6 \\
\hline \multicolumn{2}{|l|}{ Surgical procedure } \\
\hline Lobectomy & 35 \\
\hline Sublobar resection & 6 \\
\hline \multicolumn{2}{|l|}{ pStage } \\
\hline I & 30 \\
\hline II - III & 11 \\
\hline \multicolumn{2}{|l|}{ pT status } \\
\hline pT1 & 25 \\
\hline pT2-3 & 16 \\
\hline \multicolumn{2}{|l|}{$\mathrm{pN}$ status } \\
\hline pN0 & 34 \\
\hline $\mathrm{pN} 1-2$ & 7 \\
\hline \multicolumn{2}{|l|}{ CEA } \\
\hline Normal & 21 \\
\hline High & 20 \\
\hline \multicolumn{2}{|l|}{ CYFRA21-1 } \\
\hline Normal & 24 \\
\hline High & 17 \\
\hline \multicolumn{2}{|l|}{$\mathrm{SCC}$} \\
\hline Normal & 31 \\
\hline High & 10 \\
\hline \multicolumn{2}{|l|}{ KL-6 } \\
\hline Normal & 22 \\
\hline High & 19 \\
\hline
\end{tabular}

\%VC: percentage of vital capacity; UIP: usual interstitial pneumonia; CEA: carcinoembryonic antigen; CYFRA21-1: cytokeratin 19 fragments; SCC: squamous cell carcinoma-related antigen; KL-6: Krebs von den Lungen-6.
2000 and December, 2014 in our hospital. This retrospective study was approved by Medical Ethics Committee of our hospital. There were 31 men and 10 women (median age: 71 years old). The patients with died of other disease, including AE of IP, were excluded. The diagnosis of IP was confirmed based on a combination of clinical and radiological findings according to the clinical criteria proposed by the Japanese Respiratory Society [11]. Briefly, the definition of IP was made based on a clinical history of a specific form of chronic, progressive, fibrosing interstitial pneumonia that was observed on radiological imaging, and it required the exclusion of other forms of interstitial pneumonia, including medication or systematic disease. The patients were categorized into two groups according to their radiological appearance on a computed tomography (CT) scan: 1) A usual interstitial pneumonia (UIP) pattern, characterized by the presence of basal-dominant reticular opacities and the predominantly basal and subpleural distribution of honeycomb lesions, with equally sized cystic lesions of 2 - $10 \mathrm{~mm}$ in diameter with a thick wall. 2) A non-UIP pattern, characterized by the presence of basal-predominant ground glass opacities and infiltrative shadows inconsistent with a pattern of UIP. All patients received preoperative chest CT, brain magnetic resonance imaging (MRI) and positron emission tomography computed tomography-computed tomography (PETCT). Complete serum CEA, serum CYFRA21-1 and SCC levels of each patient were applied before surgery. Based on manufacturer recommendation, the following cut-offs for serum marker levels were used: CEA $5.0 \mathrm{ng} / \mathrm{mL}$, CYFA21$12.3 \mathrm{ng} / \mathrm{mL}$ and SCC $1.5 \mathrm{ng} / \mathrm{mL}$. All patients in this study were staged according to the TNM classification of the Union for International Cancer Control (the eighth edition) [12]. No patients underwent adjuvant chemotherapy because of a great risk of a critical adverse event. Follow-up information was collected directly from the outpatient clinic records or from family contact.

Overall cancer-specific survival time was calculated as the interval between surgery and death or last clinical evaluation. Cancer-specific survival was estimated by the Kaplan-Meier method and compared using the Log-rank test for univariate analysis. The hazard ratio (HR) and the $95 \%$ confidence intervals (CIs) were estimated using the Cox proportional hazard model, and the multivariate Cox model was developed using stepwise regression (backward selection) to adjust for potential confounding factors. P values of less than 0.05 were considered statistically significant. JMP (SAS Institute Inc., Cary, $\mathrm{NC}, \mathrm{USA}$ ) was used for all analyses.

\section{Results}

The clinical characteristics of patients were shown in Table 1. Among these patients, 35 patients who received lobectomy also underwent systemic mediastinal lymph nodes dissection, however those received sublobar resection did not undergo systemic mediastinal lymph nodes dissection. As shown in Figure 1, the overall 5-year cancer-specific survival was only $37.4 \%$. In patients with stage I disease, the 5-year cancer-specific survival was only $39.2 \%$. 


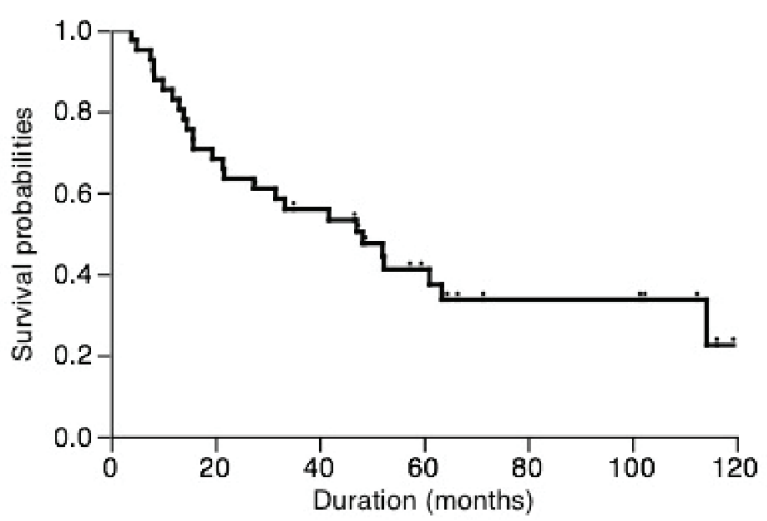

Figure 1. Overall postoperative cancer-specific survival of patients.

The 5-year cancer-specific survival based on serum CEA, CYFRA21-1 and SCC levels were shown in Figure 2. Patients with high serum CEA level had $9.4 \%$ of survival, while patients with normal CEA level had $55.6 \%$ of survival (P < 0.001 , Fig. 2a). On the other hand, the cancer-specific 5-year survival of patients with high and normal serum CYFRA21-1 levels was $25.7 \%$ and $51.6 \%$, respectively. Similarly, patients with high and normal serum SCC levels had a 5-year survival of $40.0 \%$ and $43.2 \%$, respectively. There was a trend towards an association between serum CYFRA21-1 or SCC level and cancer-specific survival but this did not reach statistical significance $(\mathrm{P}=0.068$ and 0.061 , respectively, Fig. $2 \mathrm{~b}, \mathrm{c})$.

By univariate analysis, pT status and serum CEA level were significantly associated with the 5-year cancer-specific survival while serum CYFRA21-1 and SCC levels were not (Table 2). These two clinicopathological characteristics were further investigated in multivariate analysis. As also shown in Table 2, serum CEA level was an independent factor in predicting overall postoperative cancer specific survival $(\mathrm{P}=0.001)$.

\section{Discussion}

To the best of our knowledge, this is the first report to demonstrate the prognostic role of serum CEA level in resected NSCLC patients with IP. In this study, we revealed that serum CEA level was an independent significant predictive factor for resected NSCLC patients with IP.

A multi-center study has demonstrated that resected
NSCLC patients with IP had a poor prognosis [4]. They reported that the 5-year overall survival rates were $54.2 \%$ in patients with pathological stage IA NSCLC with IP and $88.3 \%$ in those without IP [4]. Similarly, in this study, NSCLC patients with IP showed a poor prognosis.

The reason for poorer prognosis of NSCLC patients with IP has been clearly unknown in detail. The most frequently occurring NSCLC type in patients with IP is squamous cell carcinoma, whereas adenocarcinoma is the most common histologic type in patients without IP [4]. Patients with squamous cell carcinoma were speculated to have shorter survival when compared to those with adenocarcinoma [13]. The high ratio of squamous cell carcinoma histology might be one of the reasons for poor prognosis of NSCLC patients with IP, at least in part. Hata et al [14] found that the concomitant existence of IP is a risk factor for pleural invasion. They concluded that concomitant IP might cause underestimation of clinical staging, increase the chance of unexpected malignant pleurisy during surgery, and shorten survival time [14]. Therefore, it has been suggested that IP-associated NSCLC is itself a high-grade cancer.

Among NSCLC patients with IP, there are only a few studies that found the factors associated with the long-term cancerspecific survival of these patients. Sato et al [4] performed the multi-center study and demonstrated that the surgical procedure (wedge resection vs. lobectomy) and a predicted $\% \mathrm{VC}$ less than $80 \%$ were associated with survival of NSCLC patients with IP. In the present study, we failed to find the prognostic significance of the surgical procedure and $\% \mathrm{VC}$ although we did not calculate the predicted postoperative $\% \mathrm{VC}$. The reason for this discrepancy might be the small number of patients in this series. Furthermore, in this series, the numbers of patients who had $\% \mathrm{VC}$ less than $80 \%$ and received sublobar resection were only 5 and 6 , respectively. Therefore, there might be a patients' selection bias.

Among previous studies that examined the survival of patients with IP after undergoing NSCLC resection $[4,5,15$ 17], they examined the prognostic roles of clinicopathological factors, including Krebs von den Lungen-6 (KL-6), but not tumor markers. Therefore, in the present study, we examined the prognostic significance of serum tumor markers, and found that serum CEA level might serve as an efficient prognostic indicator after surgery in NSCLC with IP. On the other hand, there was a trend towards an association between serum CYFRA21-1 or SCC levels and cancer-specific survival but this did not reach statistical significance. Although the number of
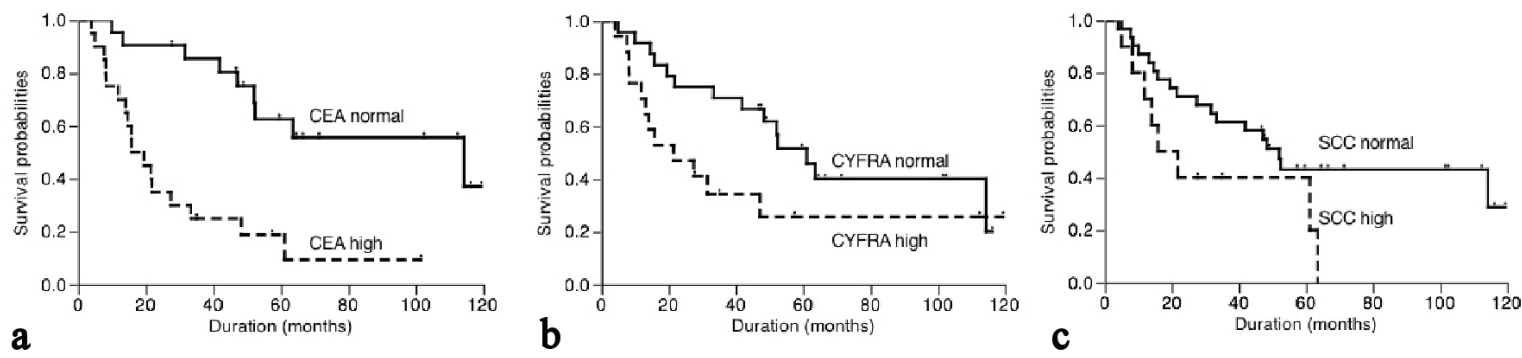

Figure 2. Postoperative cancer-specific survival of patients based on serum CEA (a), CYFRA21-1 (b) and SCC (c) levels. 
Table 2. Univariate and Multivariate Analyses

\begin{tabular}{|c|c|c|c|c|c|c|c|c|}
\hline & \multicolumn{4}{|c|}{ Univariate analysis } & \multicolumn{4}{|c|}{ Multivariate analysis } \\
\hline & \multirow{2}{*}{ Hazard ratio } & \multirow{2}{*}{ P value } & \multicolumn{2}{|c|}{$95 \% \mathrm{CI}$} & \multirow{2}{*}{ Hazard ratio } & \multirow{2}{*}{ P value } & \multicolumn{2}{|c|}{$95 \% \mathrm{CI}$} \\
\hline & & & Lower & Upper & & & Lower & Upper \\
\hline Gender & 0.695 & 0.420 & 0.254 & 1.634 & & & & \\
\hline Smoking status & 0.542 & 0.230 & 0.158 & 1.422 & & & & \\
\hline Surgical procedure & 0.452 & 0.120 & 0.187 & 1.257 & & & & \\
\hline Histology & 0.793 & 0.556 & 0.363 & 1.731 & & & & \\
\hline pT status & 0.425 & 0.035 & 0.193 & 0.939 & 0.501 & 0.092 & 0.224 & 1.122 \\
\hline $\mathrm{pN}$ status & 0.440 & 0.109 & 0.184 & 1.224 & & & & \\
\hline KL-6 & 0.522 & 0.102 & 0.236 & 1.140 & & & & \\
\hline
\end{tabular}

\%VC: percentage of vital capacity; UIP: usual interstitial pneumonia; CEA: carcinoembryonic antigen; CYFRA21-1: cytokeratin 19 fragments; SCC: squamous cell carcinoma-related antigen; KL-6: Krebs von den Lungen-6; Cl: confidence interval.

patients in this series is very small, we believe that the statistical prognostic significance of serum CEA level is meaningful. Many studies investigated the associations between serum CEA levels and the survival of NSCLC patients. Two systematic reviews showed that serum CEA level carried prognostic and predictive information on the risk of death of NSCLC patients $[6,7]$. Although underlining mechanism of the prognostic roles of CEA has been unknown in detail, CEA is a cell adhesion molecule associated with poor clinical outcomes, which is proposed to result from a variety of mechanisms, including promotion of invasion, dissemination, metastasis and immune suppression [18]. Therefore, NSCLC with IP and high serum CEA level might be more aggressive cancer, thereby worsening their prognosis and increasing the potential for the tumor to recur. Apart from NSCLC, it was reported that serum CEA level is elevated in approximately half of patients with IP and is correlated with disease severity [19]. Therefore, there is a possibility that IP-associated NSCLC had another different mechanism for high serum CEA level when compared to NSCLC without IP [19].

Our study had several limitations. It was a single-center, retrospective study with a relatively small sample size. The small samples sometimes affect statistical accuracy. Furthermore, pathological TNM staging in our series was poorer because patients received sublobar resection did not undergo systemic mediastinal lymph nodes dissection. Thus, conclusions from the present study may have a bias, indicating that multi-center studies are needed.

\section{Conclusions}

To the best of our knowledge, this is the first report to demon- strate the prognostic role of serum CEA level in patients with resected NSCLC with IP.

\section{Conflict of Interest}

The authors have declared that no conflict of interest exists.

\section{References}

1. Tanita T, Chida M, Hoshikawa Y, Handa M, Sato M, Sagawa $\mathrm{M}$, Ono S, et al. Experience with fatal interstitial pneumonia after operation for lung cancer. J Cardiovasc Surg (Torino). 2001;42(1):125-129.

2. Sato T, Teramukai S, Kondo H, Watanabe A, Ebina M, Kishi K, Fujii Y, et al. Impact and predictors of acute exacerbation of interstitial lung diseases after pulmonary resection for lung cancer. J Thorac Cardiovasc Surg. 2014;147(5):1604-1611 e11603.

3. Chida M, Kobayashi S, Karube Y, Hayama M, Tamura M, Ishihama $\mathrm{H}$, Oyaizu T. Incidence of acute exacerbation of interstitial pneumonia in operated lung cancer: institutional report and review. Ann Thorac Cardiovasc Surg. 2012;18(4):314-317.

4. Sato T, Watanabe A, Kondo H, Kanzaki M, Okubo K, Yokoi K, Matsumoto K, et al. Long-term results and predictors of survival after surgical resection of patients with lung cancer and interstitial lung diseases. J Thorac Cardiovasc Surg. 2015;149(1):64-69, 70 e61-62.

5. Kobayashi S, Matsumura Y, Karube Y, Nishihira M, Inoue T, Araki O, Maeda S, et al. Inflammation-Based Prognostic Score Predicts Postoperative Survival of Patients 
with Interstitial Pneumonia After Undergoing Lung Cancer Resection. World J Surg. 2018;42(7):2143-2152.

6. Grunnet M, Sorensen JB. Carcinoembryonic antigen (CEA) as tumor marker in lung cancer. Lung Cancer. 2012;76(2):138-143.

7. Wang XB, Li J, Han Y. Prognostic significance of preoperative serum carcinoembryonic antigen in nonsmall cell lung cancer: a meta-analysis. Tumour Biol. 2014;35(10):10105-10110.

8. Pujol JL, Molinier O, Ebert W, Daures JP, Barlesi F, Buccheri G, Paesmans M, et al. CYFRA 21-1 is a prognostic determinant in non-small-cell lung cancer: results of a meta-analysis in 2063 patients. Br J Cancer. 2004;90(11):2097-2105.

9. Niklinski J, Burzykowski T, Niklinska W, Laudanski J, Chyczewski L, Rapellino M, Furman M. Preoperative CYFRA 21-1 level as a prognostic indicator in resected nonsmall cell lung cancer. Eur Respir J. 1998;12(6):1424-1428.

10. Vassilakopoulos T, Troupis T, Sotiropoulou C, Zacharatos P, Katsaounou P, Parthenis D, Noussia O, et al. Diagnostic and prognostic significance of squamous cell carcinoma antigen in non-small cell lung cancer. Lung Cancer. 2001;32(2):137-144.

11. Raghu G, Collard HR, Egan JJ, Martinez FJ, Behr J, Brown KK, Colby TV, et al. An official ATS/ERS/JRS/ ALAT statement: idiopathic pulmonary fibrosis: evidence-based guidelines for diagnosis and management. Am J Respir Crit Care Med. 2011;183(6):788-824.

12. Bierley JD, Gospodarowicz MK, Wittekind C. TNM classification of malignant tumours. 8th ed. Wiley; 2017.

13. Nakamura H, Sakai H, Kimura H, Miyazawa T, Marush- ima H, Saji H. Difference in Postsurgical Prognostic Factors between Lung Adenocarcinoma and Squamous Cell Carcinoma. Ann Thorac Cardiovasc Surg. 2017;23(6):291-297.

14. Hata A, Suzuki H, Nakajima T, Tanaka K, Fujiwara T, Wada H, Iwata T, et al. Concomitant interstitial lung disease is a risk factor for pleural invasion in lung cancer. Ann Thorac Surg. 2017;103(3):967-974.

15. Taniguchi D, Yamasaki N, Miyazaki T, Tsuchiya T, Matsumoto K, Hatachi G, Kakugawa T, et al. The surgical outcomes of lung cancer combined with interstitial pneumonia: a single-institution report. Surg Today. 2017;47(11):1397-1404.

16. Saito Y, Kawai Y, Takahashi N, Ikeya T, Murai K, Kawabata Y, Hoshi E. Survival after surgery for pathologic stage IA non-small cell lung cancer associated with idiopathic pulmonary fibrosis. Ann Thorac Surg. 2011;92(5):18121817.

17. Sekihara K, Aokage K, Oki T, Omori T, Katsumata S, Ueda T, Miyoshi T, et al. Long-term survival after complete resection of non-small-cell lung cancer in patients with interstitial lung disease. Interact Cardiovasc Thorac Surg. 2018;26(4):638-643.

18. Beauchemin N, Arabzadeh A. Carcinoembryonic antigen-related cell adhesion molecules (CEACAMs) in cancer progression and metastasis. Cancer Metastasis Rev. 2013;32(3-4):643-671.

19. Fahim A, Crooks MG, Wilmot R, Campbell AP, Morice AH, Hart SP. Serum carcinoembryonic antigen correlates with severity of idiopathic pulmonary fibrosis. Respirology. 2012;17(8):1247-1252. 